\title{
Worm Burdens in Schistosome Infections
}

\author{
B. Gryseels and S.J. de Vlas
}

Schistosomiasis, caused by fluke worms of Schistosoma spp, is one of the most common tropical diseases. Despite decades of research and progress tonards the control of the disease', many aspects of the dynamics of infection and immunity remain unresolved. There is, in fact, not even an approximate measure of how many worms are harboured by infected humans. Epidemiological, mathematical and biomedical arguments indicate that individual worm burdens in endemic areas number hundreds to thousands of adult schistosomes, instend of the few to dozens generally assumed on the basis of awailable autopsy data. As Bruno Gryseels and Sake de Vlas here discuss, this hypothesis has important consequences for research and control, as many constants in srhistosominsis research have to be reconsitered.

In contrast to intestinal worms such as Asciris and Trichuris, which are expelled after treatment and which can then be counted, direct quantification of Schistosoma mansoni in endemic situations is impossible. In control programmes and epidemiological studies, intensity of infection is estimated indirectly by counting the number of $S$. mansoni eggs in calibrated faeces samples, which are usually converted into eggs per gramme faeces (EPG). Surprisingly little effort has been made to relate EPGs to worm numbers, and to estimate actual worm burdens in endemic situations.

Quantifi ation of worm burdens would greatly improve our understanding of the dynamics of schistosome populations, host immunity, morbidity and control. The regulation of schistosome populations is still fundamentally debated: for some authors, transmission is the main regulatory level ${ }^{1}$, for others the host ${ }^{2}$. The answer has profound implications for control: should we concentrate on destroying snails or on developing vaccines? Epidemiological models that could test the various hypotheses against ficld data depend strongly on variables representing schistosome numbers ${ }^{3-5}$.

Acquired immunity, developed over years of exposure, is believed to determine individual levels of susceptibility to (re)infection 0.7 , and vaccine development has therefore received high priority ${ }^{8,4}$. However, without a measure of worm burdens, the protective effect of a vaccine will be difficult to evaluate, especially if it would also trigger anti-fecundity immunity ${ }^{\S}$. Obviously, anti-infection and anti-fecundity effects cannot be distinguished on the basis of faecal egg counts.

Population chemotherapy has reduced morbidity in many endemic countries, so that transmission control now becomes the next objective ${ }^{(1)}$. Whether interruption or even reduction of transmission can be

\footnotetext{
Bruno Gryseels was at the Department of Parasitology and Tropical Medicine. University of Leiden. The Netherlands, and is currently at the Prince Leopold Institute of Tropical Medicine, Nationalestraat 155, B-2000 Antwerpen, Belgium. Sake de Vlas is at the Centre for Decision Sciences in Tropical Disease Control (CDTDC). Department of Public Health, Erasmus University Rotterdam, PO Box 1738, 3000 DR Rotterdam, The Netherlands. Tel: +32 3 2476200, Fax: +32 $3216 / 43$ I, e-mail: bgryseels@itg.be
}

pursued via chemotherapy or vaccination depends crucially on pre-intervention worm burdens. Indeed, if these were in the range of hundreds to thousands, even a $99 \%$ effective drug or vaccine would leave a few worms in most infected people. The remaining light infections, often not detectable by stool examination, will then act as a continued reservoir for transmission ${ }^{11}$. Without additional interventions which disturb the ccologically established balance betwe n humans and parasite populations, the latter will (after cessation of chemotherapy) inevitably re-establish their initial levels.

\section{Commonly assumed knowledge and its consequences}

The average productivity of mated female S. mansoni worms is about 300 eggs per day, of which aibout $50 \%$ will reach the faeces ${ }^{12-14}$. This assumption is based mainly on experiments in mice, hamsters, baboons and chimpanzees ${ }^{15,16}$, and - perhaps questionably extrapolated to humans. For a daily faecal production of about 150 grammes per human ${ }^{17}$, a simple calculation shows that EPG and worm pair (WP) burdens would have a 1:1 relationship. A 'common' egg count of $200 \mathrm{EPG}$ would then correspond with a burden of 200 worm pairs and at least 400 worms. Counts of 30000-40000EPG, such as seen in some highendemicity areas ${ }^{18,19}$, would at first sight imply incredible numbers of worms.

However, individual counts are subject to marked variations ${ }^{211,21}$. We have developed a model for individual variations in worm loakis ind egg counts, which, after extensive fitting, testing and validating, allows the: prediction of the corresponding distributions of WI' burdens $11,21,22$. Figure 1 shows the consequences of this model on the population level, when a 1.0EPG per WP ratio is assumed, for three typical endemic situations where we have worked over the past decade18,23. In all three villages, worm burdens over $100(>50 \mathrm{WP})$ wo ld be abundantly present. Also burdens over 1000 worms are a common phenomenon: even in the low-endemicity village of Buhandagaza, three percent of the population would harbour more than 1000 worms, and in the moderately endemic focus of Gilungws, this would be $17 \%$. In the intense-focus Makundju, eight percent of the population would harbour as many as over $1000(0)$ worms.

\section{Hard evidence}

It is quite remarkable that these consequences of handbook knowledge are in such flagrant conflict with the only available (and widely cited) direct measurement of worm burdens in humans. In 1968 , Cheever published the results of perfusion and dissection of 197 cadavers of people who had died of ditferent causes in a hospital in Salvador da Bahia, Brazil, of which 103 were infected with S. monsomi2t. Worms were collectred and counted, and faecal and tissue egg counts were determined from each cadaver. Figure 2 summarizes his findings with respect to faecal 


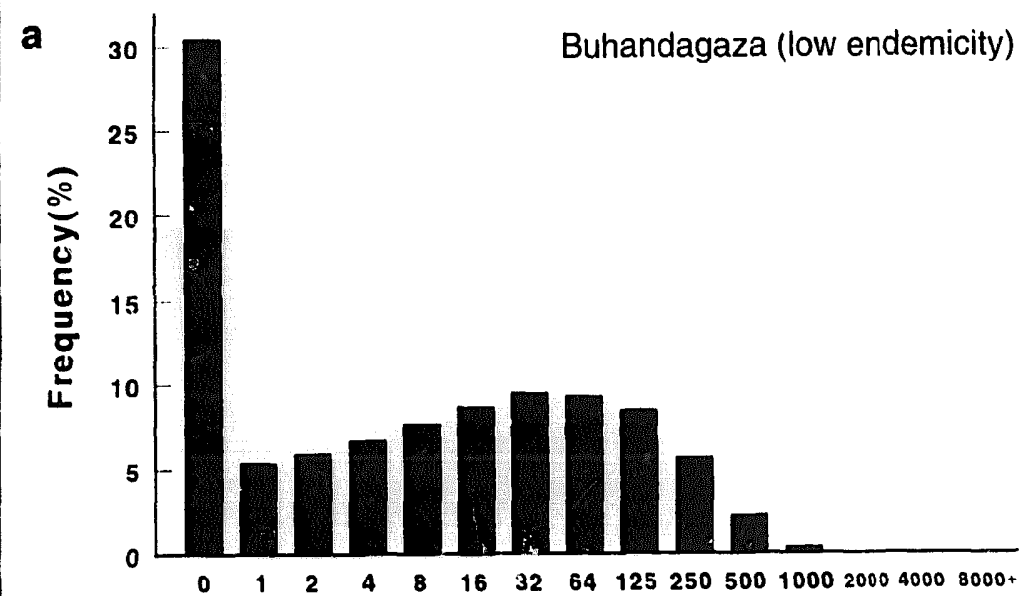

b

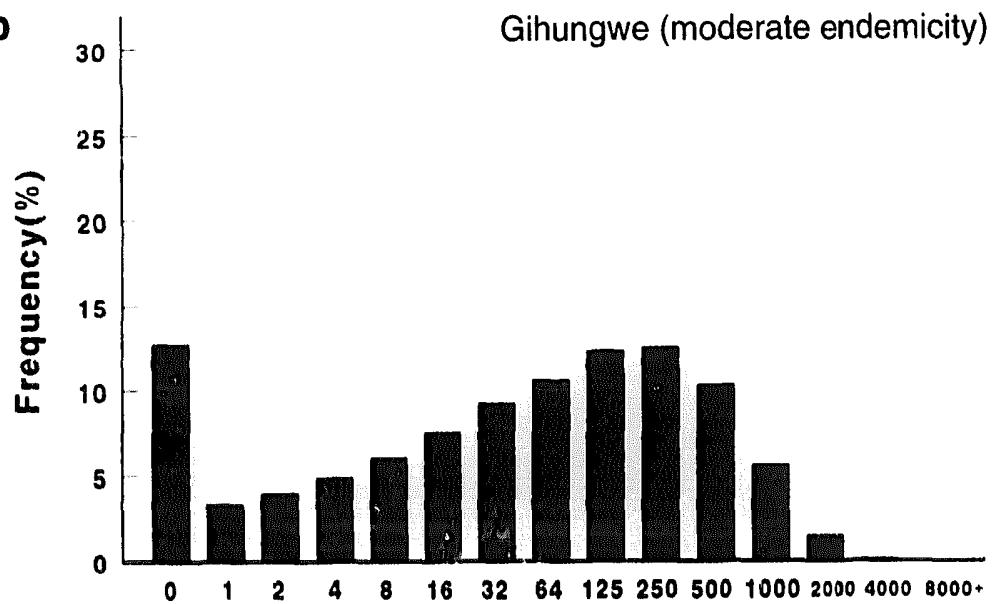

C

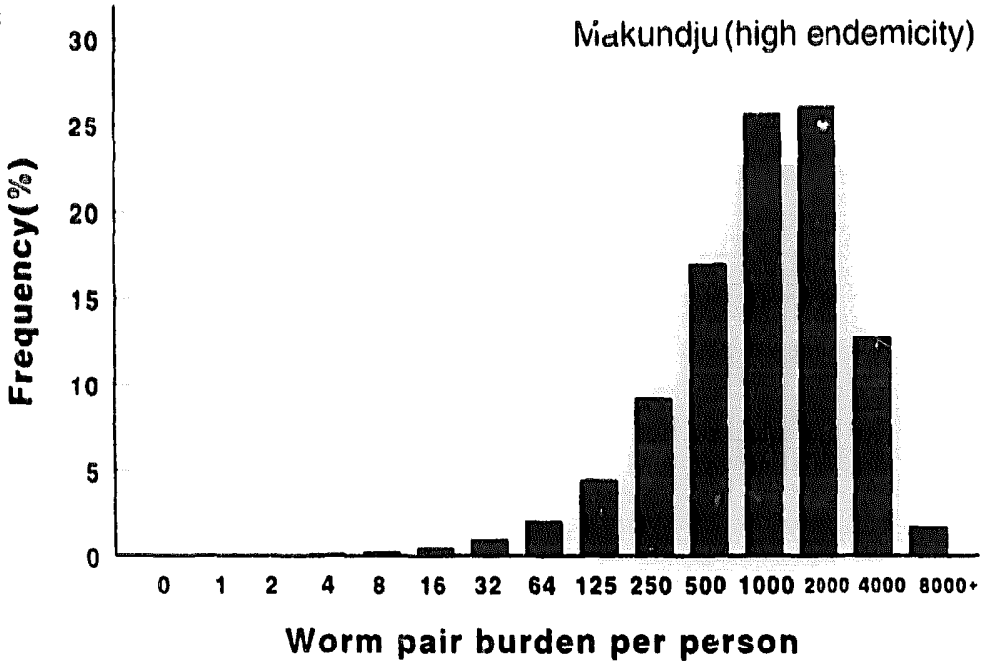

Fig. I. Predicted distribution of Schistosoma mansoni worm pairs among individuals from three different endemic communities in Burundi and Eastern Zaire: Buhandagaza. low endemicity (38\%, 80 EPG) (a); Gihungwe, moderate endemicity $(61 \%, 149 \mathrm{EPG})$ (b): and Makundju, high endemicity $(96 \%, 79 \mid$ EPG) (c). The values between parentheses represent the observed prevalences (\%) and geometric means of positive egg counts (EPG), respectively. These observations were based on $50 \mathrm{mg}$ Kato-Katz thick smears $^{35}$. On the $x$-axis, the lower limits of the predicted worm pair burden intervals are indicated. Predictions are obtained after fitting a model for egg count variation 11.21 .22 to the observed data. The distribution of individual worm pair burdens is characterized by a negative binomial distribution of total worm burdens with mean $(M)$ and aggregation parameter $(k)$, and assuming monogamous mating with a male:female ratio equal to $1: 1$. The estimated parameter values are for Buhandagaza $M=147, k=0.23$, for Gihungwe $M=561, k=0.33$ and for Makundju $M=4288, k=1.2$. egg counts and WP loads. The majority of the cases had $<100 \mathrm{WP}$. Only ten cadavers harboured $>100 \mathrm{WP}$; the highest number was 1608 (3669 worms) found in a fouryear-old child whe had died from ulcerative colitis.

Evidently, this autopsy series does not represent a normal endemic community, particularly with regard to the higher range of worm burdens. Most terminal patients belonged to older age groups, in which infections are generally less intense ${ }^{10,12-14}$. Because of their illness and hospitalization, they probably had not been exposed to transmission for a considerable time anyway. Furthermore, most subjects came from arotind Salvador, a (sub)urban area with low transmission. It is therefore safe to assume that worm burdens in more typical, rural endemic communities will be considerably higher than those reflected in Fig. 2.

Nevertheless, the autopsy studies could help to clarify the relationship between worm pairs and faecal egg counts. Figure 2 shows a significant positive correlation, although individual ratios of EPG per WP vary strongly. From the data, the overall EPG per WP ratio can be calculated to be 5.5 (with $95 \%$ contidence limits 3.9-7.9), significantly higher than the 1.() assiumed above. However, there are a number of reasoms why this autopsy relationship may not be valid in natural endemic situntions as well. First, a worm recovery rate of $100 \%$ could not possibly be achieved 24.25 ; in six cadavers with positive tissue-egg counts, for example, no worm pairs were recovered. Second, by definition the study population consisted of terminally ill hospital patients, mostly with chronic diseases, in whom faeces production is usually reduced 24 . The stools from the cadavers indeed contained less debris and required less concentration than those of live patients 24 . This factor would probably be even more important in comparison to patients from a rural endemic setting, where diets are usually rich in fibres. Third, Cheever corrected the cadaver EPG count for mushy and liquid stools with a factor 1.9 to 2.6 , a correction which is usually not made in field observations. Without this correction the ratio would be 3.4EPG per WP (with 95\% confidence limits 2.4-4.9).

The ratio of EPG per WP in normal endemic situations is thus most probably several factors lower than 
that derived from the autopsy studies. Cheever himself noted that the EPG per WP ratio in his autopsy series was unusually high, as compared to mice ${ }^{24}$. From a later autopsy study on S. haematobium patients in Egypt ${ }^{26,27}$, in which also many light infections with S. mansoni were encountered, an EPG per WP of 2.8 was calculated (without correction for stool consistency) ${ }^{21}$, but these data were much less complete than the Brazilian set ${ }^{24}$.

A complication in relating egg counts to worm numbers is that in humans with high worm loads, female worms may become iess fertile. Some authors saw evidence in the Braziliarı autopsy data for such density-dependent egg production ${ }^{28,29}$. However, they excluded nine infected individuals with zero egg counts from their analysis and based their statistical analy sis on individual EPG per WP ratios, which is a questionable approach. Their conclusion was therefore challenged by both Cheever ${ }^{25}$ and, based on a re-analysis including the Egyptian autopsy data, by Wertheimer et al..$^{30}$ Key:ner and Slater ${ }^{31}$ previously drew attention to pitfalls in deriving density dependence from parasite fecundity data in general. Figure 2 shows that, with a log-trarisformation, conditions for linear regression can be met, which is not the case with EPG per WP ratios. As the slope does not differ significantly from 1.0, density dependence cannot be confirmed from the Brazilian autopsy data. Density dependence might still exist for worm burdens higher than shown in Fig. 2, but worm loads corresponding to high EPGs would then be even higher.

\section{Anecdotal evidence}

Goldsmith it al.32 reported the results of extracorporeal blood filtration of living patients during splenectomy. Worm pair counts of the three cases (67, 383 and (69) were highly inconsistent with egg counts $(125,100$ and $1100 \mathrm{EPG}$, respectively). If anything, they show that low EPGis can be found in people with high worm burdents. Worm counts were certainly underestimated with this method, as it is unlikely that all worms were passing through the shunt or withheld by the filter mesh ${ }^{32}$.

There are very little data on the cercarial challenge an individual in an endemic community is actually confronted with. The results of the few availab! natural mice exposure experiments vary widely. In St Lucia, Sturrock ${ }^{3.3}$ found only a few infections in over 11000 naturally exposed mice, illustrating the erratic character of transmission in such low endemicity areas. In foci with continuous, intense transmission such as Makundju, quite d:ferent observations have been made. Mice exposed for a few hours in natural waters in which women and children dwclled for hours daily,

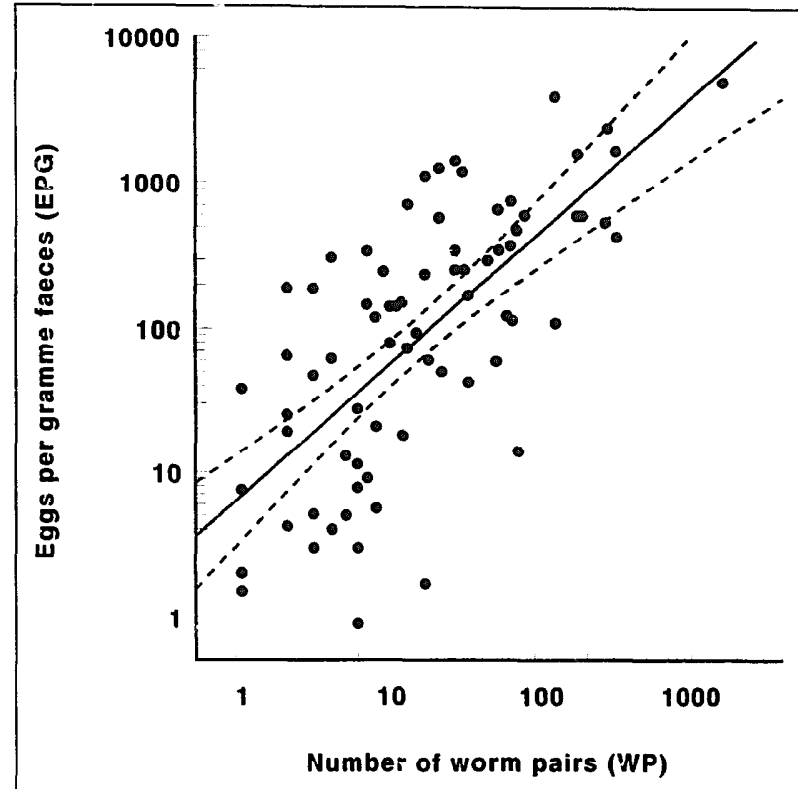

Fig. 2. Relation between eggs per gramme faeces (EPG) and number of Schistosoma mansoni worm pairs recovered by autopsy from 74 out of 103 infected cadavers described by Cheever ${ }^{24}$. In total, 29 cases were excluded for lack of egg counts (16), no worm pairs recovered (7), use of antimonial drugs before investigation (3) or incomplete autopsy (3). In nine cases, zero egg counts in infected cadavers were assigned a value of one-half the minimum number of eggs that could have been detected by the procedures used; these differ as EPGs were based on examination of variable amounts of faecal material (50-200 mg). Log-transformations of both EPGs and worm pair burdens clearly satisfy normality and homoscedasticity conditions to apply regression. The straight line represents the best fitting equation for the relacionship between number of worm pairs (WP) and EPG $=6.83$. WP0.92. with $95 \%$ confidence limir (dashed lines). The slope 0.92 suggests a slight density dependence in fecundity, but the trend is far from significant $(p>0.25)$. We can therefore calculate a single value for egg production per worm pair, EPG per WP $=5.5$ (within 95\% confidence limits 3.9-7.9) at the geometric mean of $16 \mathrm{WP}$. In the text, we discuss the representativity of these values for endemic situations.

contracted dozens to hundreds of worms (Table 1). It is thus quite probable that, in such conditions, individuals are challenged by hundreds of cercariae per day and carry thousands of worms. A simple comparison of blood volumes between humans and mice makes clear, moreover, that five WP in mice is, relatively, a heavier infection than 5000 WP in adult humans.

\section{Conclusions}

There is a discrepancy between commonly assumed EPG per WP ratios and those found in autopsy series.

Table I. Results of mice exposure and perfusion experiments in the intense focus of Makundju (Maniema, Zaire) ${ }^{a}$

Duration of
exposure (min)
16
60

120

Total no. of mice
8
8
9

Without WP
2
0
1

Mean no. of WPc
$19 \pm 11$
$82 \pm 35$
$38 \pm 27$

a Data from Ref. 18.

b Mice with severe agony or which had already died could not be properly perfused.

cThe mean number of worm pairs (WP) recovered from only those mice that harboured at least one worm pair (within $95 \%$ confidence limits) is indicated. The maximum number of worms recovered was 344 in one hour. 
Table 2. Predicted range in Schistosoma mansoni worm pair (WP) burden for a given egg per gramme faeces (:FG) count in three different endemic situations"

\begin{tabular}{rccc}
\hline EPG & $\begin{array}{c}\text { Buhandagaza } \\
\text { (low endemicity) }\end{array}$ & $\begin{array}{c}\text { Gihungwe } \\
\text { (moderate endemicity) }\end{array}$ & $\begin{array}{c}\text { Makundju } \\
\text { (high endemicity) } \\
11-2977\end{array}$ \\
100 & $0-72$ & $0-192$ & $83-3553$ \\
400 & $22-427$ & $28-863$ & $222-4220$ \\
1000 & $86-727$ & $108-1423$ & $422-4947$ \\
10000 & $183-1032$ & $238-1977$ & $1972-8778$ \\
\hline
\end{tabular}

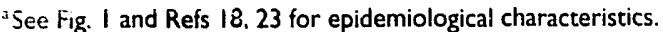

'Indicated are $90 \%$ ranges; ie. $5 \%$ chance that WP is below and $5 \%$ that WP is above this range. The calculations are based on a model for egg count variation 11.21 .22 , assuming a productivity of 1.0 EPG per WP.

There are good reasons to believe that the EPG per WP ratio of about $1: 1$ is a realistic starting point. This would imply that endemic worm counts are much higher than generally assumed (Fig. 1).

However, egg counts cannot straightforwardly be translated into worm burdens. The extensive withinindividual variation in schistosome egg counts ${ }^{21,21}$ results in a wide range of corresponding worm burdens. Furthermore, this range is dependent on the characteristics of the endemic situation. In an area of low endemicity, a high EPG is likely to be due to an incidental peak in egg excretion of this individual, whereas in an intense focus a high egg count more probably reflects a genuine high worm burden. Based on the model outlined above, we have calculated the range of worm burdens corresponding to given EPGs in different endemic situations (Table 2). In a lowendemicity community such as Buhandagaza, the possible range corresponding with 1000)EPG has $1000 \mathrm{WP}$ as an upper limit. However, in a highendemicity village such as Makundju 1(0)() EPG may reflect up to 50(0) WP. On the other hand, in such an intense focus, negative egg counts most likely correspond to positive worm burdens. Of course, these predictions assume satisfaction of modelling conditions, like perfect negative binomial distributions of egg counts and worm burdens, and no disturbances by, for example, migration or chemotherapy interventions? 21

There are good reasons to believe that worm numbers in endemic communities are significantly higher than is usually perceived. Clearly, fundamental questions in the dynamics of schistosome infections remain unanswered, hampering the development of epidemiological models, and consequently of tools and strategies for control. Hopefully, new techniques for the assessment of worm burdens, such as antigen detection assays ${ }^{34}$, will improve our diagnostic and epidemiological toolbox. Meanwhile, we have to resort to statistical and mathematical approaches to solve, or at least to identify, such crucial gaps in our knowledge.

\section{References}

I Warren, K.S. (1973) Regulation of the prevalence and intensity of schistosomiasis in man: immunology or ecology? I. Infoct. Dis. $127,369-373$

2 Bradley, D.J. (19/2) Regulation of parasite populations: a general theory of the epidemiology and control of parusitic infections. Trams R. Soc. Trop. Metl. Hyg. 66, 697-708

3 Woolhouse, M.E.J. (1991) On the application of mathematical models of schistosome transmission dynamics, I: natural transmission. Acta Trop. 49, 241-270

4 Woolhouse, M.E.J. (1992) On the application of mathematical models of schistosome transmission dynamics, II: control Acto Trop. 50, 189-204

5 Gryseels, B. Uncertainties in the epidemiology and control of schistosomiasis and the contribution of modeling. Am. J. Trop. Mit. Hus. (in press)

6 Hagan, $P$. (1942) Reinfection, exposure and immunity in human schistosomiasis. Parasitoloyy Today \&, 12-16

7 Maizels, R.M. it al. (1993) Immunological modulation and evasion by helminth parasites in human populations. Noture 365 , $797-815$

8 Capron, A. it al. (1994) Development of a vaccine strategy against human and bovine schistosomiasis: background and upidate. Trop. Gerogr. Mlid. 46, 242-246

9) Bergejuist, N.R., Hall, B.F. and James, S. (1994) Schistomiasis vaccine development: translating basic research into practical results. Immmmologist 2, 131-1.34

10) World Health Organization (1993) The control of schistosomiasis: second report of the WHO expert committee. WHO Tich. Rip. Sir. No. 830)

11 De Vlas, S.J. and Grvseels, B. (1992) Underestimation of Schistosoma mansoni prevalences. Parasifolog!! Tothly 8 , $27 \cdot+277$

.2 Rollinson, D. and Simpuon, A.J.C. (eds) (1987) The Biolngy of

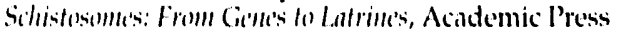

1.3 Jorctan, I., Webbe, C. and Sturrock, R.F. (eds) (1999) Ilumm sidnstusimminsis, CAB intermational

It World I lealth (Organization (198.5) The control of schistosomiasis: report of a WHO expert committee. WIK) Tirk. Ri'p. Sir.

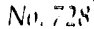

15 loker, I:S. (148,3) A comparative study of the life-histories of

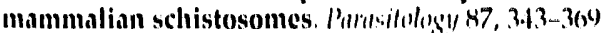

In Che'ver, A.W. it al. (I)(4) Kinetics of egg production and egg excretion by Schistosoma mansoni and $S$. juponicum in mice infected with a single pair of worms. Am. I. Trop. Med. I/ys. 50, $281-295$

17 Brooks, F.P. (1985) in Bist and Tinylor's Mhysiglogical Btsis of Meritial Prititice, 11 th ededn (West J.B., ed.), pp $6.34-790)$, Williams $\&$ Wilkins

Is Grysecls, B. and Polderman, A.M. (1987) The morbidity of schistosomiasis in Maniema (Zaire). Tram: R. Sox. Trop. Mort. Hys. $81,2(12-21) 4$

19 Stelma, F.F. it al. (i)ur) Epidemiology of Schistosoma mamsoni infection in a recently exposed community in Northern Senegal. Aill. I. Tripl. Miti. Hus. 49, 7011-71)6

20) Barreto, M.L. it al. (1978) Stability of faecal egg excretion in Schistosoma mansomi infection. Trms. $R$. Soli. Trop. Mol. Hys. $72,181-187$

21 De Vlas, S.J. it al. (1942) A model for variations in single and repeated egg counts in Schistosoma mansoni infections. Parnsitolegy 104, 451-460)

32 De Vlas, S.J., Van Oortmarssen, G.J. and Gryscels, B. (1992) Validation of a model for variations in Schistosom! munsoni egg count:s. Trums. R. Six. Trop. Mid. Hus. 86, 645

23 Gryseels, B., Nkulikyinka, L. and Engels, D. (1991) Repeated community-based chemotherapy for the control of Schistosoma mansoni: effect of screening and selective treatment in prevalences and intensities of infection. Am. I. Trop. Meit. Hy/s. 45, 509-517

24 Cheever, A.W. (1968) A quantitative post-mortem study of schistosomiasis mansonit in man. Am. J. Trop. Mct. Hy/3. 17 38-64

25 Cheever, A.W. (1986) Density-dependent fecundity in Schistosoma mansoni infections in man: a reply. Trants. $R$. Soc. Trop. 
Med. Hyg. 80, 991-992

26 Kamel, I.A. et al. (1977) Schistosoma mansoni and S. haematobium infections in Egypt, I: evaluation of techniques for recovery of worms and eggs at necropsy. Am. J. Trop. Me'd. Hys. 26, 696-701

27 Cheever, A.W. et al. (1977) Schistasoma mansoni and S. haematobiun infections in Egypt, II: quantitative parasitological findings at necropsy. Amt. J. Trop. Met. Hyg. 26, 702-716

28 Anderson, R.M. and May, R.M. (1982) Population dynamics of human helminth infections: control by chemotherapy. Natur' 297, 557-563

29 Medley, G. and Anderson, R.M. (1985) Density dependent fecundity in Schistosoma mansoni in man. Trans. $R$. Soc. Trop. Med. Hyg. 79, 532-534

30 Wertheimer, S.P. ('t al. (1987) Lack of demonstrable densitydependent fecundity of schistosomiasis mansoni: analysis of
Egyptian quantitative human autopsies. Am. J. Trop. Med. Hys. $37,79-84$

31 Keymer, A.E. and Slater, A.F.G. (1987) Helminth fecundity: density dependence or statistical illusion? Parasitology Today 3, 56-58

32 Goldsmith, E.I. et al. (1967) Surgical recovery of schistosomes from the portal blood. I. Am. Med. Assoc. 199, 235-240

33 Sturrock, R.F. (1973) Field studies on the transmission of Schistosoma mansoni and on the bionomics of its intermediate host, Biomplialaria glabrata, on St Lucia, West Indies. Int. J. Parasital. 3, 175-194

34 Deelder, A.M. 't al. (1994) Quantitative diagnosis of Schistosoma infections by measurement of circulating antigens in serum and urine. Trop. G'ogr. Met. 46, 233-238

35 Katz, N., Chaves, A. and Pellegrino, J. (1972) A simple device for quantitative stool thick-smear technique in schistosomiasis mansoni. Re' '. Inst. Med. Trop. Sao Paulo 14, 397-400

\title{
Schistosomes and Serpins: A Complex Busine,s
}

\author{
J. Modha, M.C. Roberts and J.R. Kusel
}

The vicue of the schistosome host-parasite relationship has changed in the past two decades. Prezionsly, it was thought the parasite simply defended itself in the face of a hostile host envirommeml. Howereer, it is now renlized that the host-parasite interaction is much more of a dynumic interplay, zohere the parasite is able to cxploit host home'ostatic me'chanisms for survizal, maturity and transmission. Here', lay Modha, Clare Roberts and fohn Kusel discuss the recent identification of serinc protense inhibitors (serpins) on the schistosome surface and sugge'st hone their propertic's might be exploited by the pormsite.

Proteases play a key role in the schistosome life cycle, being involved in penetration of host skin, parasite mutrition, immune evasion and egg excretion 1,2. Mammalian hosts, in defence ayainst the threat of uncontrulled proteolytic activity, posisess serine protease inhibitor's (serpins), whose function it is to inactivate not only stray, excess and/or redundant proteolytic activity from endogenous sources, but also that from exogenous sources (eg. from parasites and microorganisms) 3?

\section{Serpin identification and properties}

A comparison of the properties and features of schistosome-associated serpins is given in Table 1. Blanton of al. ${ }^{5}$ have screened a Schistosoma haematobiul! cDNA library with species-specific human antisera and have identified a serpin (SHSPI) with a native molecular mass of $54-58 \mathrm{kDa}$. They also report a similar molecule in S. mansonii. Ghendler et al." on the other hand, have purified Smpi56, a $56 \mathrm{kDa}$ serpin, from adult $S$. mansoni worm extracts. In addition, contrapsin ${ }^{7}$ has been identified in homogenates ${ }^{8}$ and on the surface" of $S$. mnntsoni worms from mice. Contrapsin is also present in homogenates of $S$. jnponicum and S. bovis worms ${ }^{8}$, but whether this is true of Smpi56

Jay Modha, Clare Roberts and John Kusel are at the Division of Biochemistry and Molecular Biology. The Davidson Building, University of Glasgow. UK G12 8QQ. Tel: +44 I4I 330 462I, Fax: +44 141 330 4620, e-mail: gbca66@udcf.gla.ac.uk and SHSPI remains to be determined. The presence of contrapsin and Smpi56 in S. haematobium has not been reported. As far as stage specificity is concerned, all three serpins are present in or on the adult worm, but their origin seems to be different. Recombinant DNAand metabolic-labelling techniques show that SHSPI and Smpi56, respectively, are synthesized by the schistosome. Contrapsin, however, may be acquired from host blood, since its forms in adult worm homogenates and in mouse plasma share immunological identity". Uptake of host molecules is a common feature of $S$. mansoni, and contrapsin may be added to the catalogue of other host-derived molecules associated with the parasitel(1-14.

\section{Distribution and complexes}

The distribution of serpins on the worm surface is also varied. Anti-contrapsin antibodies label the surface (but not the tubercles) and subtegumental cells intensely, whereas anti-SHSPI antibodies localize on the spines and bind only weakly to the rest of the worm surface. SHSPI appears to be an integral membrane protein and is not reported to be present in subtegumental cells ${ }^{5}$, but contrapsin is present in the aqueous phase of a Triton X-114 fractionation", indicating that it is unlikely to be an integral membrane protein ${ }^{15}$. The distribution of Smpi56 is not described, although it is present in tegumental extracts". All three serpins are present in complex form, either on the woin surface, or in tegumental extracts, but the molecules complexing with the serpins have not been identified in all cases. Undoubtedly, the cognate protease for Smpi56 is the $28 \mathrm{kDa}$ schistosome elastase ${ }^{10}$ (G.R. Newport, Abstract*), but the worm molecules complexing with contrapsin and SHSPI remain to be identified. The inhibition of complex formation of contrapsin following pretreatment of worm homogenate with phenylmethyl-sulphonyl fluoride and

\footnotetext{
* Sichistosome elastases: biological importance, structure, function and stage-specific expression. Biochemical Society Symposium No. 53. PP $1 ! 5-121.1987$
} 